University of Windsor

Scholarship at UWindsor

1973

\title{
Photon-scattering theory of the quenching of hydrogenic metastables
}

Gordon W. F. Drake

University of Windsor

R. B. Grimley

Follow this and additional works at: https://scholar.uwindsor.ca/physicspub

Part of the Physics Commons

\section{Recommended Citation}

Drake, Gordon W. F. and Grimley, R. B.. (1973). Photon-scattering theory of the quenching of hydrogenic metastables. Physical Review A, 8 (1), 157-160.

https://scholar.uwindsor.ca/physicspub/109

This Article is brought to you for free and open access by the Department of Physics at Scholarship at UWindsor. It has been accepted for inclusion in Physics Publications by an authorized administrator of Scholarship at UWindsor. For more information, please contact scholarship@uwindsor.ca. 


\title{
Photon-Scattering Theory of the Quenching of Hydrogenic Metastables
}

\author{
G. W. F. Drake and R. B. Grimley \\ Department of Physics, University of Windsor, Windsor, Ontario, Canada \\ (Received 13 February 1973)
}

\begin{abstract}
The quenching of hydrogenic metastables is formulated as a near-resonant photon-scattering problem, and a complete analysis of the polarization and angular distribution of the emitted radiation is presented. The contribution from $n p$ states with $n>2$ is calculated, and an alternative method of measuring the Lamb shift is suggested.
\end{abstract}

\section{INTRODUCTION}

Precise theoretical calculations are now available for the $2 s_{1 / 2}-2 p_{1 / 2}$ Lamb shift of hydrogenic ions up to large values of the nuclear charge $Z .^{1}$ In several recent experiments, ${ }^{2-5}$ the Lamb shift for hydrogenic ions such as $\mathrm{Li}^{2+}, \mathrm{C}^{5+}$, and $\mathrm{O}^{7+}$ has been deduced from the measured electric field quench rate of the metastable $2 s_{1 / 2}$ state. The well-known Bethe-Lamb (BL) phenomenological quenching theory ${ }^{6}$ with corrections for mixing with the $2 p_{3 / 2}$ state $^{2.7}$ is used to interpret the measurements. The BL theory is quite adequate for low values of $Z$, but relativistic corrections and contributions from the $n p_{1 / 2,3 / 2}$ states with $n>2$ became increasingly important with increasing $Z$ and should eventually be included in making precision comparisons between theoretical and experimental Lamb shifts in highly ionized atoms. In this paper, the emission of quench radiation is regarded as a near-resonant photon-scattering process and the contribution from $n p$ states with $n>2$ is calculated. In addition, a complete analysis of the polarization and angular distribution of the quench radiation is presented, and an alternative method of measuring the Lamb shift is suggested.

\section{THEORY}

The quenching of a metastable hydrogenic ion by a static electric field can be regarded as the zero-frequency limit of two-photon-scattering processes with a resonance near zero frequency. The theory then closely resembles that for other resonant scattering phenomena near a level crossing, such as the Hanle effect. The static electric field may be thought of as a beam of very-low-frequency photons polarized in the external field direction. If there are $N_{1}$ incident photons of frequency $\omega_{1}$ and polarization $\hat{e}_{1}$ per unit volume, then the differential transition rate for the emission of a photon of frequency $\omega_{2}^{ \pm}=E_{i}-E_{f} \pm \omega_{1}$ and polarization $\hat{e}_{2}$ into solid angle $d \Omega_{2}$ is given in the non- relativistic electric-dipole approximation by

$\frac{d \omega_{i f}}{d \Omega_{2}}=\frac{N_{1} \omega_{1}}{c^{3}}\left[\left|Q\left(\omega_{1}, \omega_{2}^{-}\right)\right|^{2}\left(\omega_{2}^{-}\right)^{3}+\left|Q\left(-\omega_{1}, \omega_{2}^{+}\right)\right|^{2}\left(\omega_{2}^{+}\right)^{3}\right]$,

where

$Q\left(\omega_{1}, \omega_{2}\right)=e^{2} \sum_{n}\left(\frac{\left(\overrightarrow{\mathrm{r}} \cdot \hat{e}_{2}\right)_{f n}\left(\overrightarrow{\mathrm{r}} \cdot \hat{e}_{1}\right)_{n i}}{E_{n}-E_{i}+\omega_{1}}+\frac{\left(\overrightarrow{\mathrm{r}} \cdot \hat{e}_{1}\right)_{f n}\left(\overrightarrow{\mathrm{r}} \cdot \hat{e}_{2}\right)_{n i}}{E_{n}-E_{i}+\omega_{2}}\right)$

in atomic units.

The first term of (1) corresponds to singly stimulated two-photon emission and the second term to anti-Stokes Raman scattering. In analogy with resonant scattering theories, the energies $E_{n}$ are understood to include the radiation shifts $\Delta E_{n}$ and level widths $\Gamma_{n}$ so that $E_{n}=E_{n}^{0}+\Delta E_{n}-\frac{1}{2} i \Gamma_{n}$. In the limit of zero frequency, the two terms of (1) contribute coherently and should be added before squaring. Also $8 \pi N_{1} \omega_{1} \rightarrow F^{2}$ as $\omega_{1} \rightarrow 0$, where $F$ is the electric field strength.

For the $2 s_{1 / 2}-1 s_{1 / 2}$ transition of hydrogen, the summation over intermediate states can be divided into a contribution from the $n p_{1 / 2}$ states and a contribution from the $n p_{3 / 2}$ states. After averaging over the $2 s_{1 / 2}$-state degeneracy and summing over the $1 s_{1 / 2}$-state degeneracy, the differential transition rate is proportional to the sum of terms in (1) of the form

$$
\begin{aligned}
\left|\bar{Q}\left(\omega_{1}, \omega_{2}\right)\right|^{2} & =\frac{1}{2} \sum_{m_{i}, m_{f}}\left|Q\left(\omega_{1}, \omega_{2}\right)\right|^{2} \\
& =\frac{1}{9}\left|\hat{e}_{1} \cdot \hat{e}_{2}\right|^{2}|A|^{2}+\frac{1}{9}\left|\hat{e}_{1} \times \hat{e}_{2}\right|^{2}\left|A^{\prime}\right|^{2},
\end{aligned}
$$

with

$$
\begin{aligned}
& A=A_{1 / 2}\left(\omega_{1}\right)+A_{1 / 2}\left(\omega_{2}\right)+2 A_{3 / 2}\left(\omega_{1}\right)+2 A_{3 / 2}\left(\omega_{2}\right), \\
& A^{\prime}=A_{1 / 2}\left(\omega_{1}\right)-A_{1 / 2}\left(\omega_{2}\right)-A_{3 / 2}\left(\omega_{1}\right)+A_{3 / 2}\left(\omega_{2}\right),
\end{aligned}
$$

and

$$
A_{J}(\omega)=e^{2} \sum_{n} \frac{\langle 1 s|z| n p\rangle\langle n p|z| 2 s\rangle}{E_{n p_{J}}-E_{2 s_{1 / 2}}+\omega}
$$

for $J=\frac{1}{2}$ and $J=\frac{3}{2}$. The matrix elements in (6) are 
the simple hydrogenic transition integrals without spin coupling. The first term of (3) specifies the same angular and frequency dependence as for the $2{ }^{1} S_{0}-1{ }^{1} S_{0}$ transition of helium, while the second term is the same as that for the $2{ }^{3} S_{1}-1{ }^{1} S_{0}$ twophoton transition of helium. ${ }^{8}$ In common with the helium results, $A$ is a maximum and $A^{\prime}=0$ when $\omega_{1}=\omega_{2}$. For large frequencies $\omega_{1} \neq \omega_{2}, A^{\prime}$ is much smaller than $A$, but for frequencies $\omega_{1} \leqslant \Delta E_{L}$, where $\Delta E_{L}$ is the Lamb shift, $A^{\prime}$ and $A$ are of roughly equal magnitude.

Equation (3) completely specifies the statistical relationship between the polarization vectors of the two photons. Each polarization vector can take two linearly independent directions perpendicular to the direction of propagation. For example, if the polarization $P$ is observed in the plane perpendicular to $\hat{e}_{1}\left(\hat{e}_{1}\right.$ is the electric field direction in a quenching experiment), then it follows immediately from (3) that

$$
P=\frac{I_{\|}-I_{\perp}}{I_{\|}+I_{\perp}}=\frac{|A|^{2}-\left|A^{\prime}\right|^{2}}{|A|^{2}+\left|A^{\prime}\right|^{2}},
$$

where $I_{\|}$and $I_{\perp}$ are the emitted intensities polarized parallel and perpendicular to $\hat{e}_{1}$. The above is in agreement with the result derived by Ott, Kauppila, and Fite, ${ }^{9}$ except for the contribution from states of higher $n$.

If Eq. (3) is summed over the two linearly independent directions for photon 2 , then

$$
\begin{aligned}
\sum_{\hat{e}_{2}}\left|\bar{Q}\left(\omega_{1}, \omega_{2}\right)\right|^{2}= & \frac{1}{9}|A|^{2}\left(a_{\perp}^{2}+a_{\|}^{2} \cos ^{2} \theta\right) \\
& +\frac{1}{9}\left|A^{\prime}\right|^{2}\left[a_{\perp}^{2}+a_{\|}^{2}\left(1+\sin ^{2} \theta\right)\right],
\end{aligned}
$$

where $a_{\perp}$ and $a_{\|}$are the polarization vector amplitudes for photon 1 perpendicular and parallel to the scattering plane and $\theta$ is the angle between the two-photon propagation vectors. For the static field quenching case, assume for definiteness that an electric field in the $z$ direction is produced by a polarized low-frequency photon beam in the $x$ direction and the quench radiation summed over both polarizations is observed in the $y z$ plane. Then $\theta=\frac{1}{2} \pi, a_{\|}=\cos \varphi$, and $a_{\perp}=\sin \varphi$, where $\varphi$ is the angle between the direction of observation and the electric field direction, and ( 8 ) becomes

$\sum_{\hat{e}_{2}}\left|\bar{Q}\left(\omega_{1}, \omega_{2}\right)\right|^{2}=\frac{1}{9}\left(|A|^{2}+\left|A^{\prime}\right|^{2}\right) \cos ^{2} \varphi+\frac{2}{9}\left|A^{\prime}\right|^{2} \sin ^{2} \varphi$.

The result depends only on $\varphi$ and is invariant with respect to rotations of the observation direction about the electric field direction. Defining $I_{T} \|$ and $I_{T \perp}$ to be the total intensities per unit solid angle emitted in directions parallel and perpendicular to the electric field, then the anisotropy factor $R=\left(I_{T \|}-I_{T \perp}\right) /\left(I_{T \|}+I_{T \perp}\right)$ is

$$
R=\frac{\left|A^{\prime}\right|^{2}-|A|^{2}}{3\left|A^{\prime}\right|^{2}+|A|^{2}}
$$

If the Lamb shift vanishes, then $A^{\prime} \rightarrow A$ and $R \rightarrow 0$. The value of $R$ is independent of the field strength, provided that higher-order perturbation corrections are negligible. Since a measurement of $R$ involves only counting the total numbers of photons emitted in the parallel and perpendicular directions, it may provide a convenient method of obtaining Lamb shifts. No time resolution is required provided that all intensity components decay with the same time constant.

Finally, averaging over $\hat{e}_{1}$ and summing over $\hat{e}_{2}$ results in

$$
\begin{aligned}
\frac{1}{2} \sum_{\hat{e}_{1}, \hat{e}_{2}}\left|\bar{Q}\left(\omega_{1}, \omega_{2}\right)\right|^{2}= & \frac{1}{18}|A|^{2}\left(1+\cos ^{2} \theta\right) \\
& +\frac{1}{18}\left|A^{\prime}\right|^{2}\left(3-\cos ^{2} \theta\right),
\end{aligned}
$$

which is the factor correlating the directions of the photon propagation vectors if neither polarization is observed. Integration over angles yields

$$
\begin{aligned}
\left|\bar{Q}\left(\omega_{1}, \omega_{2}\right)\right|_{\mathrm{av}}{ }^{2} & =\int d \Omega \frac{1}{2} \sum_{\hat{e}_{1}, \hat{e}_{2}}\left|\bar{Q}\left(\omega_{1}, \omega_{2}\right)\right|^{2} \\
& =\frac{1}{27} \pi\left(|A|^{2}+2\left|A^{\prime}\right|^{2}\right) .
\end{aligned}
$$

In the limit of zero frequency for photon 1, the quench rate is given in terms of the field strength $F$ by

$$
w_{i f}=\frac{4 \omega_{2}^{3} F^{2}}{27 c^{3}}\left(|A|^{2}+2\left|A^{\prime}\right|^{2}\right) .
$$

For the Stark quenching of hydrogen, the terms involving $\omega_{2}$ in (4) and (5) are very small and the quench rate is well approximated by

$$
w_{i f}=\frac{4 \omega_{2}^{3} F^{2}}{9 c^{3}}\left[\left|A_{1 / 2}(0)\right|^{2}+2\left|A_{3 / 2}(0)\right|^{2}\right],
$$

in agreement with the $\mathrm{BL}$ result when only the $n=2$ term is retained in the summation over intermediate $n p$ states in (6). The cross terms between the $2 p_{1 / 2}$ and $2 p_{3 / 2}$ states vanish when averaged over polarizations and directions of emission. ${ }^{7}$ On the other hand, if all the $n p_{1 / 2}$ and $n p_{3 / 2}$ states are taken to be degenerate, then $A^{\prime}=0$ and $A=3\left[A_{1 / 2}\left(\omega_{1}\right)+A_{1 / 2}\left(\omega_{2}\right)\right]$. The quench rate given by (13) is then formally identical with that derived previously for the $1 s 2 s^{1} S_{0}$ state of helium. ${ }^{10}$

The spontaneous two-photon decay rate is

$$
d w\left(\omega_{1}, \omega_{2}\right)=\frac{1}{(2 \pi)^{3} c^{6}}\left|Q\left(\omega_{1}, \omega_{2}\right)\right|^{2}\left(\omega_{1} \omega_{2}\right)^{3} d \omega_{1} d \Omega_{1} d \Omega_{2} .
$$

Using the foregoing results to sum over both 
polarization vectors and integrate over angles, the averaged decay rate reduces to

$d w\left(\omega_{1}, \omega_{2}\right)=\frac{8}{27 \pi c^{6}}\left(\omega_{1} \omega_{2}\right)^{3}\left(|A|^{2}+2\left|A^{\prime}\right|^{2}\right) d \omega_{1}$

per unit frequency interval. The contribution from the $A^{\prime}$ term, although small for small values of $Z$, has not been included in calculations of the two-photon decay rate. ${ }^{11}$ However, a careful analysis is required since the relativistic and retardation effects are of equal importance.

\section{CONTRIBUTION FROM HIGHER $n p$ STATES}

To a good approximation, we can take the $n p_{1 / 2}$ and $n p_{3 / 2}$ states with $n>2$ to be degenerate and write

$$
\begin{aligned}
& A_{J}(0)=\frac{e^{2}\langle 1 s|z| 2 p\rangle\langle 2 p|z| 2 s\rangle}{E_{2 p_{J}}-E_{2 s_{1 / 2}}}+B, \\
& A_{J}\left(E_{2 s_{1 / 2}}-E_{1 s_{1 / 2}}\right)=C,
\end{aligned}
$$

where

$$
\begin{aligned}
& B=e^{2} \sum_{n=3}^{\infty} \frac{\langle 1 s|z| n p\rangle\langle n p|z| 2 s\rangle}{E_{n p}-E_{2 s}}, \\
& C=e^{2} \sum_{n=2}^{\infty} \frac{\langle 1 s|z| n p\rangle\langle n p|z| 2 s\rangle}{E_{n p}-E_{1 s}} .
\end{aligned}
$$

The above equations give the values of $A_{J}\left(\omega_{1}\right)$ and $A_{J}\left(\omega_{2}\right)$ evaluated at $\omega_{1}=0$ and $\omega_{2}=E_{2 s_{1 / 2}}-E_{1 s_{1 / 2}}$. The quantities $A$ and $A^{\prime}$ are then calculated from (4) and (5). The BL results are obtained by setting $B=0$ and $C=0$.

The summations over intermediate states can be calculated by the implicit summation technique introduced by Schwartz and Tieman ${ }^{12,13}$ and utilized by Zernik. ${ }^{14}$ This technique requires the numerical integration of a first-order differential equation. An alternative technique capable of very high accuracy is to represent the complete set of $n p$ states (including the continuum) by a discrete Sturmian basis set generated by diagonalizing the Hamiltonian $H$ in a basis set constructed from functions of the form

$$
\psi_{n}=\sum_{i=1}^{N} a_{i}^{(n)} r^{i} e^{-r / 2} Y_{1}^{0}(\hat{r}) .
$$

The $a_{i}^{(n)}$ are determined by the conditions

$$
\begin{aligned}
& \left\langle\psi_{m} \mid \psi_{n}\right\rangle=\delta_{m n}, \\
& \left\langle\psi_{m}|H| \psi_{n}\right\rangle=\epsilon_{n} \delta_{m n} .
\end{aligned}
$$

One of the $\psi_{n}$ is then the exact $2 p$ eigenfunction, while the remaining $N-1$ linearly independent functions form a variational approximation to the orthogonal complement in Hilbert space. The re- sults obtained in this way with a ten-term basis set are

$$
B=12.415592 Z^{-4} \text { a.u. , } C=-3.476366 Z^{-4} \text { a.u. }
$$

correct to the figures quoted. The choice of phases is such that $\langle 1 s|z| 2 p\rangle\langle 2 p|z| 2 s\rangle=-2^{7}(\sqrt{2}) / 3^{4}$. As a check, the same basis set yields the nonrelativistic spontaneous two-photon decay rate $w_{i f}=8.2292 Z^{6} \mathrm{~s}^{-1}$, in exact agreement with Klarsfeld' $s^{11}$ result when the revised value of $\alpha^{-1}=137.03602$ is used. Since the first term of (17) decreases as $Z^{-6}$, the corrections $B$ and $C$ become increasingly important with increasing nuclear charge. For example, at $Z=20$, the quantities $P$ and $R$ in (7) and (9) are decreased by about $0.1 \%$, and the total quench rate (13) increased by about $0.005 \%$. Approximate values of $P$ and $R$ calculated from the energy levels tabulated by Garcia and $\mathrm{Mack}^{15}$ are given in Table I.

The calculation presented here is entirely nonrelativistic, except for the phenomenological introduction of the Lamb shift and level width. There are further important relativistic and retardation effects which will be discussed in a future publication. The additional small corrections due to hyperfine structure ${ }^{16}$ for nuclei with nonzero spin should also be considered.

An experiment to measure the anisotropy $R$ would involve passing a beam of hydrogenic metastable ions through an electric field as in conventional quenching experiments, and measuring the

TABLE I. Values of the polarization $P$ and anisotropy $R$. These values were calculated with the $2 s_{1 / 2}-2 p_{1 / 2}$ and $2 s_{1 / 2}-2 p_{3 / 2}$ energy differences tabulated by Garcia and Mack (Ref. 15), and $\Gamma(2 p)=4.699 \times 10^{8} \mathrm{Z}^{4} \mathrm{~s}^{-1}$.

\begin{tabular}{rcc}
\hline$Z$ & $P$ & $R$ \\
\hline 1 & -0.32862 & 0.14112 \\
2 & -0.26744 & 0.11795 \\
3 & -0.23252 & 0.10415 \\
4 & -0.20988 & 0.09497 \\
5 & -0.19206 & 0.08762 \\
6 & -0.17811 & 0.08177 \\
7 & -0.16487 & 0.07616 \\
8 & -0.15616 & 0.07242 \\
9 & -0.14760 & 0.06873 \\
10 & -0.14087 & 0.06580 \\
11 & -0.13436 & 0.06295 \\
12 & -0.12873 & 0.06047 \\
13 & -0.12331 & 0.05807 \\
14 & -0.11868 & 0.05602 \\
15 & -0.11414 & 0.05399 \\
16 & -0.11006 & 0.05216 \\
17 & -0.10631 & 0.05047 \\
18 & -0.10270 & 0.04884 \\
19 & -0.10264 & 0.04882 \\
20 & -0.09919 & 0.04725 \\
\hline \hline
\end{tabular}


total emitted intensities through identical slits in the parallel and perpendicular directions. Rotation of the field direction through $90^{\circ}$ relative to the slits and photon counters would eliminate many systematic errors. Since $R$ is independent of field strength in lowest order, nonuniformities in the field strength are not important, provided that the field direction remains well defined throughout the observation region. A small uncertainty $\delta R$ in the measured value of $R$ produces an uncertainty $\delta\left(\Delta E_{L}\right)$ in the Lamb shift given approximately by $\delta\left(\Delta E_{L}\right) / \Delta E_{L} \simeq \delta R / R$. It would also be of interest to verify that both the parallel and perpendicular intensity components decay with the same time constant.

\section{ACKNOWLEDGMENTS}

Financial support by the National Research Council of Canada is gratefully acknowledged. One of us (R. B.G.) is the holder of a NRC Bursary.
${ }^{1}$ G. W. Erickson, Phys. Rev. Lett. 27, 780 (1971).

${ }^{2}$ C. Y. Fan, M. Garcia-Munoz, and S. A. Sellin, Phys. Rev. 161, 6 (1967).

${ }^{3}$ M. Leventhal and D. E. Murnick, Phys. Rev. Lett. 25, 1237 (1970).

${ }^{4}$ D. E. Murnick, M. Leventhal, and H. W. Kugel, Phys. Rev. Lett. 27, 1625 (1971).

${ }^{5}$ H. W. Kugel, M. Leventhal, and D. E. Murnick, Phys. Rev. A 6, 1306 (1972).

${ }^{6}$ W. E. Lamb, Jr. and R. C. Retherford, Phys. Rev. 79, 549 (1950).

${ }^{7}$ H. K. Holt and S. A. Sellin, Phys. Rev. A 6, 508 (1972)

${ }^{8}$ G. W. F. Drake, G. A. Victor, and A. Dalgarno, Phys. Rev.
180, 25 (1969).

${ }^{9}$ W. R. Ott, W. E. Kauppila, and W. L. Fite, Phys. Rev. A 1, 1089 (1970)

${ }^{10}$ G. W. F. Drake, Can. J. Phys. 50, 1896 (1972).

${ }^{11}$ S. Klarsfeld, Phys. Lett. 30, 382 (1969), and earlier references therein.

${ }^{12}$ C. Schwartz, Ann. Phys. (N.Y.) 6, 156 (1959).

${ }^{13}$ C. Schwartz and T. J. Tieman, Ann. Phys. (N.Y.) 6, 178 (1959).

${ }^{14}$ W. Zernik, Phys. Rev. 133, 117 (1964).

${ }^{15}$ J. D. Garcia and J. E. Mack, J. Opt. Soc. Am. 55, 654 (1965).

${ }^{16}$ J. S. Casalese and E. Gerjuoy, Phys. Rev. 180, 327 (1969).

\title{
Calculation of Atomic and Molecular Momentum Expectation Values and Total Energies from Compton-Scattering Data
}

\author{
Irving R. Epstein \\ Department of Chemistry, Brandeis University, Waltham, Massachusetts 02154
}

(Received 8 February 1973)

\begin{abstract}
Measurement of a system's Compton profile makes possible, in principle, the calculation of its electronic radial momentum distribution. From this quantity, the expectation values $\left\langle p^{n}\right\rangle$ and the total energy may be obtained. The feasibility of using Compton-scattering data in this way is examined in view of the limitations imposed by (i) random experimental errors on the measured profile, and (ii) restriction of the profile data to a finite range of momenta. It is concluded that while present techniques may be adequate for a few systems, improved experimental methods are required before Compton scattering becomes a useful source of atomic and molecular energies.
\end{abstract}

\section{INTRODUCTION}

Recent years have seen a tremendous revival of interest in Compton scattering as a tool for studying electronic momentum distributions in atoms, molecules, and solids. ${ }^{1}$ Significant experimental advances have occured in both $\mathrm{x}-\mathrm{ray}^{2.3}$ and $\gamma-$ ray $^{4,5}$ techniques. Compton profiles over a broad range of momenta and accurate to a fraction of a percent at the peak may now be obtained in a matter of days, even for relatively heavy elements. ${ }^{5}$
Compton-scattering measurements yield, at least in principle, a full one-dimensional (or, in the case of anisotropic systems, three-dimensional) momentum distribution for the scatterer. It should therefore be possible to calculate from Compton data expectation values of operators which are functions of momentum. In particular, one should be able to obtain the quantities

$$
\left\langle p^{n}\right\rangle=\int_{0}^{\infty} p^{n} I(p) d p
$$

